\title{
Femoral morphometry and femur length in mice selected for different body conformations. A potential animal model suitable for QTLs mapping
}

\begin{abstract}
Four lines of mice derived from the $\mathrm{CBi}$ stock, selected for different body conformations (CBi-, low body weight - short tail; $\mathrm{CBi}^{+}$, high body weight - long tail; $\mathrm{CBi} / \mathrm{L}$, low body weight - long tail; $\mathrm{CBi} / \mathrm{C}$, high body weight short tail), differ in the biomass sustained per unit of skeleton weight. Femur length was modified in response to artificial selection either for high or low skeleton length. This feature suggests that these lines could be discriminated using the morphometric profile of their femurs. The femurs were obtained from both sexes at 15 weeks of age. A total of 16 measurements were taken on each bone. Genotype and gender effects for almost all measurements $(\mathrm{P}<0.001)$ were seen. Genotype $\mathrm{x}$ gender interactions $(\mathrm{P}<0.05)$ for some length measurements were also found. For sexual dimorphic characters, males had wider and shorter femurs than females. The results of principal components and discriminant analysis showed that the morphometric profile of the femur is a reliable and accurate means of identifying these inbred strains of mice as all female and male animals were assigned to the correct genotype. When the reciprocal hybrids among these genotypes were performed different responses in femur length were observed. So, the underlying genetic differences to this phenotypic differentiation emerge, at least partially, as a consequence of the exploitation of different sources of genetic variation for the trait in each selective procedure, jointly with the effect of simultaneously acting dispersive processes suggesting the potential usefulness of these genotypes as an animal model suitable for the identification of QTLs associated with femur growth.
\end{abstract}

Key Words: bone morphometry; femur length; body conformation; artificial selection; mice

\section{Zusammenfassung}

Titel der Arbeit: Femurmorphometrie und Femurlänge bei nach unterschiedlichen Körperproportionen ausgewählten Mäusen. Ein potenzielles Tiermodell für die QTLs Kartierung

Vier CBi Mäuselinien mit unterschiedlichen Körperproportionen (CBi-; niedriges Körpergewicht - kurzer Schwanz; CBi+, hohes Körpergewicht - langer Schwanz; CBi/L, niedriges Körpergewicht - langer Schwanz; CBi/C, hohes Körpergewicht - kurzer Schwanz) wurden für die Untersuchungen ausgewählt, Sie unterschieden sich hinsichtlich der auf das Skelettgewicht bezogenen Biomasse. Die Femurlänge der Tiere war, als Ergebnis einer Selektion nach hoher oder niedriger Skelettlänge, unterschiedlich. Als Folge zeigte sich, dass diese Linien sich durch ihre Femurmorphologie unterschieden. Die Erfassung der Femurlänge erfolgte bei beiden Geschlechtern im Alter von 15 Wochen. An jedem Knochen wurden 16 Maße genommen. Es ergaben sich signifikante $(\mathrm{P}<0.001)$ Genotyp- und Geschlechtereffekte. Ebenso konnten Genotyp x Geschlechter Interaktionen für einige Längenmaße nachgewiesen werden $(\mathrm{P}<0.05)$. Hinsichtlich des Geschlechtsdimorphismus zeigten sich beim Femur bei den männlichen Mäusen breitere und kürzere Formen als bei den weiblichen Mäusen. Im Ergebnis der Hauptkomponenten- und Diskriminanzanalyse ergab sich, dass das morphometrische Profil des Femurs eine zuverlässige und genaue Möglichkeit zur Identifikation des Mäusestammes bietet, weil danach alle männlichen und weiblichen Tiere den richtigen Genotypen zugeordnet wurden. Wenn die reziproken Hybriden innerhalb der Genotypen betrachtet wurden, ergaben sich hinsichtlich der Femurlänge unterschiedliche Ergebnisse. Die bei verschiedenen Genotypen zu beobachtenden phänotypischen Unterschiede sind zumindest teilweise als das Ergebnis der unterschiedlichen Nutzung der genetischen Varianz der Merkmale bei den einzelnen Selektionsschritten anzusehen. Es wird vorgeschlagen, dass durch die gleichzeitigen simultanen Verteilungsprozesse, diese Genotypen in Verbindung mit dem Femurwachstum als ein geeignetes Tiermodell für die Identifizierung von QTLs genutzt werden können.

Schlüsselwörter: Knochenmorphometrie, Femurlänge, Körperproportion, künstliche Selektion, Maus 


\section{Introduction}

One of the main objectives of the genetic analysis of animal growth in general, and of bone growth in particular, is to elucidate the genetic architecture of the related traits under study (ZENG et al., 1999). That means knowing the number of loci affecting a trait, their chromosomal location, the magnitude of their phenotypic effects, their allelic frequencies and the types of gene action involved in their expression. Several strategies have been developed to identify and characterize genes involved in the regulation of mouse growth which could be briefly summarized in long-term artificial selection experiments, the study of single gene mutations producing major phenotypic changes, targeted gene deletions and transgenics, and QTLs (quantitative trait loci) characterization (CORVA and MEDRANO, 2001; MIELENZ and SCHÜLER, 2002; BÜNGER et al., 2005). Although the production of transgenic and knockout animals requires the previous knowledge of the gene associated with the phenotype under study, to integrate that gene in a recipient animal or to replace the functional allele by a null one producing a loss-of-function phenotype, respectively, artificial selection and QTL methodology work with anonymous genes underlying the phenotypic variance of complex quantitative traits (BROCKMANN et al., 1996).

The development of molecular techniques and genetic maps based on DNA markers by one side and of the appropriate statistical tools by the other, have enhanced our ability to study the genetic basis of quantitative variation. Loci affecting quantitative traits could be mapped in animal models using crosses of specific inbred lines (FISLER and WARDEN, 1997) or crosses between outbred lines (TALBOT et al., 1999) or between lines derived from long-term selection experiments (HALEY et al., 1994; DAS et al., 1996; ROSOCHACKI et al., 2005). Because this approach requires the analysis of the pedigree resulting from crossing extreme individuals, the availability of genetically divergent strains and a linkage map covering all of the genome are limitative resources (BÜNGER et al., 2002).

In bone research the use of the femur is widely spread. At first sight, the different lines of mice derived from the $\mathrm{CBi}$ stock herein studied, obtained as the result of a longterm selection experiment for different body conformations (HINRICHSEN et al., 1999), appear to be an useful biological resource for studying the genetical basis of bone growth as they exhibited significant differences in several skeletal traits (DI MASSO et al., 1991; 1997b; 1998) and also in bone biomechanics (DI MASSO et al., 1997a) and muscle-bone relationships (DI MASSO et al., 2004). The femur length, for example, was modified in response to selection pressure for either high or low skeletal length but this response was only evident when that change was compatible with the function of the skeleton as a scaffold for the soft tissues (WALTER, et al., 1993).

Therefore, genotypes selected for long (skeleton) tail enlarged their femurs irrespective if they were simultaneously selected for either high or low body weight, whereas those lines selected for short (skeleton) tail only shortened their femurs when they were selected for low body weight as this bone enlarged in $\mathrm{CBi} / \mathrm{C}$ mice selected for high body weight.

The objective of the present study was to characterize four lines of mice derived from the $\mathrm{CBi}$ stock selected for different body conformations jointly with the unselected control line. This characterization was first done in terms of the morphometric profile of their femurs by means of a multivariate approach to investigate if artificial selection had been successful in differentiating them, and second, in terms of the source of genetic variance probably exploited in each selective criterion, evaluating by this way 
their potential usefulness for the identification of QTLs associated with femur growth.

\section{Materials and Methods}

Mice

Four lines of mice $(\mathrm{CBi}-, \mathrm{CBi} / \mathrm{L}, \mathrm{CBi} / \mathrm{C}, \mathrm{CBi}+)$ divergently selected for different body conformations by means of a quantitative index which combines body weight and tail length at 49 days of age, and the unselected control line (CBi) were used. Two lines were generated favouring the positive genetic correlation between both traits (agonistic selection: $\mathrm{CBi}-$, low body weight - short tail; $\mathrm{CBi}+$, high body weight - long tail), whereas the other two were originated by selecting against the aforementioned association (antagonistic selection: $\mathrm{CBi} / \mathrm{L}$, low body weight - long tail; $\mathrm{CBi} / \mathrm{C}$, high body weight - short tail). Lines were inbred by limiting the population size being their average inbreeding coefficient approximately 0.985 .

Experiment I - Femur morphometric profile

Mice were randomly sampled from litters of eight to ten animals and contemporaneously reared in groups of six gender - matched companions, in polypropylene cages (32 x $24 \times 10 \mathrm{~cm}$ ) with wood shavings for bedding. They were kept in the same mouse room under the same breeding conditions $\left(23 \pm 1^{\circ} \mathrm{C}\right.$, on a $12-$ hour-on /12-hour-off light cycle) and received the same diet of mouse food (Cargill Laboratory Chow, pelletized) and water ad libitum. Femurs were obtained from both female and male mice ( $\mathrm{n}=10$ individuals per genotype-sex group) of 120 days of age and prepared according to the method described by FESTING (1972) for the mandible. Briefly, mice were sacrificed by ether overexposure, and each right femur was excised and carefully cleaned by hand to remove all the adhering soft tissues. A total of 16 measurements (LOVELL and JOHNSON, 1983) (Figure) were taken on each femur using a standardized photographical procedure.

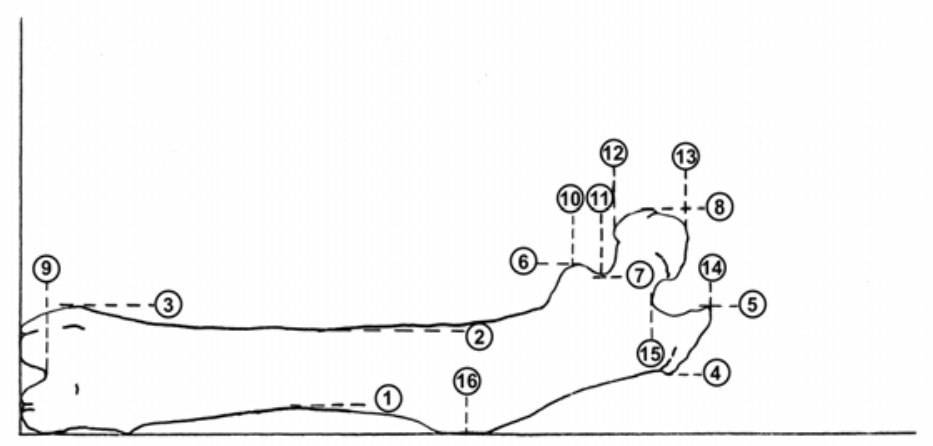

Figure: Diagram of the right femur showing the sixteen measurements made on each bone in this study (Femur measurements X1-X8: height from the $\mathrm{X}$ axis to the horizontal dotted line at each site; X9-X16: length from the $\mathrm{Y}$ axis to the vertical dotted line at each site) (16 Messungen am rechten Femur, 1-8 Höhe ausgehend von der Y Achse, 9-16 ausgehend von der X Achse)

Experiment II - Femur length in reciprocal crosses

Total femur length (mm) from the greatest trochanter to the medial condyle was measured at 150 days of age in 14 male and 14 female mice from four selected lines and reciprocal crosses between them. Besides the four parental lines $(\mathrm{CBi}-, \mathrm{CBi}+$, $\mathrm{CBi} / \mathrm{L}$ and $\mathrm{CBi} / \mathrm{C}$ ), the following genetic groups were defined: (a) crosses between 
agonistically selected lines ( $\mathrm{CBi}-\mathrm{x} \mathrm{CBi}+)$ and $(\mathrm{CBi}+x$ CBi-); (b) crosses between antagonistically selected lines $(\mathrm{CBi} / \mathrm{C} \times \mathrm{CBi} / \mathrm{L})$ and $(\mathrm{CBi} / \mathrm{L} \times \mathrm{CBi} / \mathrm{C}) ;(\mathrm{c})$ crosses between lines selected for low body weight ( $\mathrm{CBi}-\mathrm{x} \mathrm{CBi} / \mathrm{L})$ and $(\mathrm{CBi} / \mathrm{L} \times \mathrm{CBi}-)$; (d) crosses between lines selected for high body weight $(\mathrm{CBi}+\mathrm{x} \mathrm{CBi} / \mathrm{C})$ and $(\mathrm{CBi} / \mathrm{C} \mathrm{x}$ $\mathrm{CBi}+$ ); (e) crosses between lines selected for short tail (skeleton) length (CBi- $\mathrm{x}$ $\mathrm{CBi} / \mathrm{C}$ ) and ( $\mathrm{CBi} / \mathrm{C} \times \mathrm{CBi}-)$; (f) crosses between lines selected for long tail (skeleton) length $(\mathrm{CBi}+\times \mathrm{CBi} / \mathrm{L})$ and $\left(\mathrm{CBi} / \mathrm{L} \times \mathrm{CBi}^{+}\right)$. In all cases the first line denotes the maternal genotype.

Heterosis was measured relative to the average of the parental lines and thus it refers to any significant departure from additivity in crossbred populations (SHERIDAN, 1981).

Heterosis estimates for femur length were calculated as follows:

Heterosis $(\%)=[($ reciprocal hybrids mean / parental lines mean $)-1]$ x 100

Statistical analysis

Experiment I - The effects of genotype, gender and (genotype $\mathrm{x}$ gender) interaction were evaluated using a two-way analysis of variance (SOKAL and ROHLF, 1969). Data were also analysed by the multivariate techniques of principal components (PCA) and discriminant analysis (TATSUOKA, 1971).

Table 1

Mean \pm standard error $(\mathrm{mm})$ for each femur measurement $(\mathrm{M})$ in male mice (Mittelwert und Standardfehler (mm) der Femurmaße männlicher Tiere)

\begin{tabular}{|c|c|c|c|c|c|}
\hline \multirow[t]{2}{*}{$\mathrm{M}$} & \multicolumn{5}{|c|}{ Genotype } \\
\hline & CBi- & $\mathrm{CBi} / \mathrm{L}$ & $\mathrm{CBi}$ & $\mathrm{CBi} / \mathrm{C}$ & $\mathrm{CBi}+$ \\
\hline \multirow{2}{*}{1} & 0.547 & 0.895 & 0.607 & 0.729 & 0.539 \\
\hline & \pm 0.116 & \pm 0.214 & \pm 0.075 & \pm 0.065 & \pm 0.088 \\
\hline \multirow{2}{*}{2} & 2.179 & 3.019 & 2.773 & 3.088 & 2.635 \\
\hline & \pm 0.186 & \pm 0.251 & \pm 0.115 & \pm 0.162 & \pm 0.173 \\
\hline \multirow{2}{*}{3} & 2.859 & 3.566 & 3.212 & 3.434 & 3.231 \\
\hline & \pm 0.182 & \pm 0.251 & \pm 0.082 & \pm 0.115 & \pm 0.219 \\
\hline \multirow{2}{*}{4} & 0.851 & 1.771 & 1.604 & 1.718 & 1.282 \\
\hline & \pm 0.262 & \pm 0.251 & \pm 0.188 & \pm 0.235 & \pm 0.202 \\
\hline \multirow{2}{*}{5} & 2.185 & 3.509 & 3.256 & 3.405 & 2.733 \\
\hline & \pm 0.425 & \pm 0.259 & \pm 0.201 & \pm 0.226 & \pm 0.197 \\
\hline \multirow{2}{*}{6} & 3.467 & 4.402 & 4.095 & 4.452 & 3.797 \\
\hline & \pm 0.023 & \pm 0.219 & \pm 0.325 & \pm 0.452 & \pm 0.186 \\
\hline \multirow{2}{*}{7} & 3.004 & 4.156 & 3.844 & 4.122 & 3.567 \\
\hline & \pm 0.264 & \pm 0.176 & \pm 0.222 & \pm 0.336 & \pm 0.111 \\
\hline \multirow{2}{*}{8} & 4.092 & 5.734 & 5.393 & 5.632 & 5.102 \\
\hline & \pm 0.343 & \pm 0.268 & \pm 0.173 & \pm 0.315 & \pm 0.128 \\
\hline \multirow{2}{*}{9} & 0.402 & 0.334 & 0.352 & 0.354 & 0.397 \\
\hline & \pm 0.132 & \pm 0.089 & \pm 0.074 & \pm 0.098 & \pm 0.064 \\
\hline \multirow{2}{*}{10} & 12.147 & 13.640 & 12.969 & 13.469 & 14.809 \\
\hline & \pm 0.202 & \pm 0.305 & \pm 0.323 & \pm 0.335 & \pm 0.214 \\
\hline \multirow{2}{*}{11} & 12.677 & 14.076 & 13.397 & 13.891 & 15.302 \\
\hline & \pm 0.156 & \pm 0.262 & \pm 0.283 & \pm 0.293 & \pm 0.211 \\
\hline \multirow{2}{*}{12} & 12.958 & 14.442 & 13.739 & 14.256 & 15.762 \\
\hline & \pm 0.191 & \pm 0.286 & \pm 0.256 & \pm 0.231 & \pm 0.275 \\
\hline \multirow{2}{*}{13} & 14.240 & 16.096 & 15.356 & 15.839 & 17.361 \\
\hline & \pm 0.355 & \pm 0.314 & \pm 0.266 & \pm 0.249 & \pm 0.312 \\
\hline \multirow{2}{*}{14} & 14.874 & 17.085 & 16.016 & 16.665 & 18.083 \\
\hline & \pm 0.252 & \pm 0.346 & \pm 0.294 & \pm 0.319 & \pm 0.302 \\
\hline \multirow{2}{*}{15} & 13.653 & 15.509 & 14.658 & 15.266 & 16.689 \\
\hline & \pm 0.191 & \pm 0.357 & \pm 0.329 & \pm 0.312 & \pm 0.232 \\
\hline \multirow{2}{*}{16} & 9.992 & 11.439 & 10.355 & 11.007 & 11.993 \\
\hline & \pm 0.169 & \pm 0.233 & \pm 0.276 & \pm 0.275 & \pm 0.435 \\
\hline
\end{tabular}


Experiment II - The heterotic effect in each reciprocal cross was assessed from the statistical significance of the interaction in a $2 \times 2$ factorial experiment (2 maternal genotypes x 2 paternal genotypes).

\section{Experiment I}

\section{Results}

Means \pm SEM of femur measurements for males and females are respectively presented in Tables 1 and 2.

Table 2

Mean \pm standard error (mm) for each femur measurement $(\mathrm{M})$ in female mice (Mittelwert und Standardfehler (mm) der Femurmaße weiblicher Tiere)

\begin{tabular}{|c|c|c|c|c|c|}
\hline \multirow[t]{2}{*}{ M } & \multicolumn{5}{|c|}{ Genotype } \\
\hline & CBi- & $\mathrm{CBi} / \mathrm{L}$ & $\mathrm{CBi}$ & $\mathrm{CBi} / \mathrm{C}$ & $\mathrm{CBi}+$ \\
\hline \multirow{2}{*}{1} & 0.321 & 0.668 & 0.499 & 0.642 & 0.435 \\
\hline & \pm 0.105 & \pm 0.045 & \pm 0.097 & \pm 0.045 & \pm 0.035 \\
\hline \multirow{2}{*}{2} & 1.823 & 2.581 & 2.292 & 2.798 & 2.351 \\
\hline & \pm 0.135 & \pm 0.113 & \pm 0.164 & \pm 0.136 & \pm 0.124 \\
\hline \multirow{2}{*}{3} & 2.735 & 3.375 & 3.215 & 3.496 & 3.263 \\
\hline & \pm 0.106 & \pm 0.136 & \pm 0.177 & \pm 0.129 & \pm 0.195 \\
\hline \multirow{2}{*}{4} & 0.388 & 1.258 & 0.911 & 1.376 & 0.803 \\
\hline & \pm 0.194 & \pm 0.123 & \pm 0.268 & \pm 0.205 & \pm 0.191 \\
\hline \multirow{2}{*}{5} & 1.551 & 3.055 & 2.366 & 2.931 & 2.189 \\
\hline & \pm 0.238 & \pm 0.199 & \pm 0.349 & \pm 0.212 & \pm 0.355 \\
\hline \multirow{2}{*}{6} & 3.148 & 3.867 & 3.398 & 3.944 & 3.451 \\
\hline & \pm 0.166 & \pm 0.401 & \pm 0.255 & \pm 0.288 & \pm 0.369 \\
\hline \multirow{2}{*}{7} & 2.519 & 3.588 & 3.081 & 3.603 & 3.066 \\
\hline & \pm 0.121 & \pm 0.296 & \pm 0.173 & \pm 0.146 & \pm 0.213 \\
\hline \multirow{2}{*}{8} & 3.658 & 5.126 & 4.533 & 5.079 & 4.562 \\
\hline & \pm 0.215 & \pm 0.183 & \pm 0.236 & \pm 0.111 & \pm 0.255 \\
\hline \multirow{2}{*}{9} & 0.315 & 0.353 & 0.322 & 0.358 & 0.342 \\
\hline & \pm 0.076 & \pm 0.075 & \pm 0.033 & \pm 0.048 & \pm 0.098 \\
\hline \multirow{2}{*}{10} & 13.312 & 14.474 & 13.595 & 13.775 & 15.012 \\
\hline & \pm 0.469 & \pm 0.393 & \pm 0.366 & \pm 0.226 & \pm 0.462 \\
\hline \multirow{2}{*}{11} & 13.759 & 14.218 & 14.001 & 14.185 & 15.515 \\
\hline & \pm 0.485 & \pm 0.295 & \pm 0.325 & \pm 0.257 & \pm 0.368 \\
\hline \multirow{2}{*}{12} & 14.071 & 15.249 & 14.402 & 14.591 & 15.947 \\
\hline & \pm 0.499 & \pm 0.385 & \pm 0.314 & \pm 0.299 & \pm 0.427 \\
\hline \multirow{2}{*}{13} & 15.438 & 16.839 & 15.933 & 16.141 & 17.498 \\
\hline & \pm 0.538 & \pm 0.423 & \pm 0.365 & \pm 0.282 & \pm 0441 \\
\hline \multirow{2}{*}{14} & 15.935 & 17.786 & 16.458 & 16.891 & 17.983 \\
\hline & \pm 0.592 & \pm 0.472 & \pm 0.457 & \pm 0.389 & \pm 0.562 \\
\hline \multirow{2}{*}{15} & 14.702 & 16.268 & 15.223 & 15.498 & 16.721 \\
\hline & \pm 0.512 & \pm 0.381 & \pm 0.379 & \pm 0.243 & \pm 0.529 \\
\hline \multirow{2}{*}{16} & 10.548 & 11.905 & 10.698 & 11.168 & 12.323 \\
\hline & \pm 0.427 & \pm 0.367 & \pm 0.349 & \pm 0.215 & \pm 0.429 \\
\hline
\end{tabular}

The ANOVA analysis showed a genotype effect $(\mathrm{P}<0.001)$ for all measurements except M 9, a gender effect for all measurements $(\mathrm{P}<0.001)$ except M3 and M9 and genotype $x$ gender interactions $(P<0.05)$ for some length measurements $(M 10, M 12$, M13, M14, M15 and M16). For sexual dimorphic characters, males showed wider (M1 to M8) and shorter (M10 to M16) femurs than females in agreement with LOVELL and JOHNSON (1983).

The results of principal component analysis applied to femur measurements in males and females can be seen in Table 3. 
Table 3

Eigenvectors of the two first principal components (PC1 and PC2) in male (M) and female (F) mice selected for body conformation (Eigenvektor für die ersten zwei Hauptkomponenten (PC) bei Mäusen beider Geschlechter nach Selektion auf Körperproportion)

\begin{tabular}{llccc} 
& & & & Females \\
& PC1 & PC2 & PC1 & 0.296 \\
X1 & -0.177 & 0.293 & -0.224 & 0.239 \\
X2 & -0.277 & 0.231 & -0.279 & 0.140 \\
X3 & -0.272 & 0.141 & -0.261 & 0.295 \\
X4 & -0.258 & 0.273 & -0.251 & 0.265 \\
X5 & -0.252 & 0.289 & -0.259 & 0.199 \\
X6 & -0.236 & 0.269 & -0.222 & 0.228 \\
X7 & -0.273 & 0.241 & -0.283 & 0.199 \\
X8 & -0.292 & 0.202 & -0.299 & -0.200 \\
X9 & -0.100 & -0.047 & -0.105 & -0.320 \\
X10 & -0.263 & -0.289 & -0.249 & -0.245 \\
X11 & -0.254 & -0.311 & -0.128 & -0.327 \\
X12 & -0.255 & -0.309 & -0.251 & -0.296 \\
X13 & -0.268 & -0.279 & -0.265 & -0.229 \\
X14 & -0.284 & -0.239 & -0.288 & -0.267 \\
X15 & -0.274 & -0.267 & -0.276 & -0.258 \\
X16 & -0.215 & -0.167 & -0.269 & \\
\hline
\end{tabular}

Table 4

Genotype-gender group assignment by means of a discriminant analysis (Genotyp-Geschlechterzuordnung durch die Diskriminanzanalyse)

\begin{tabular}{|c|c|c|c|c|c|c|c|}
\hline \multirow[t]{2}{*}{$\begin{array}{c}\text { True } \\
\text { genotype }\end{array}$} & \multirow[t]{2}{*}{ Gender } & \multicolumn{5}{|c|}{ Assigned genotype } & \multirow[t]{2}{*}{ Total } \\
\hline & & CBi- & $\mathrm{CBi} / \mathrm{L}$ & $\mathrm{CBi}$ & $\mathrm{CBi} / \mathrm{C}$ & $\mathrm{CBi}+$ & \\
\hline \multirow{2}{*}{$\begin{array}{c}\text { CBi- } \\
\text { Low body weight } \\
\text { Short tail }\end{array}$} & M & 10 & & & & & 10 \\
\hline & $\mathrm{F}$ & 10 & & & & & 10 \\
\hline \multirow{2}{*}{$\begin{array}{c}\mathrm{CBi} / \mathrm{L} \\
\text { Low body weight } \\
\text { Long tail }\end{array}$} & M & & 10 & & & & 10 \\
\hline & $\mathrm{F}$ & & 10 & & & & 10 \\
\hline \multirow{2}{*}{$\begin{array}{c}\mathrm{CBi} \\
\text { unselected control }\end{array}$} & $\mathrm{M}$ & & & 10 & & & 10 \\
\hline & $\mathrm{F}$ & & & 10 & & & 10 \\
\hline \multirow{2}{*}{$\begin{array}{c}\mathrm{CBi} / \mathrm{C} \\
\text { High body weight } \\
\text { Short tail }\end{array}$} & M & & & & 10 & & 10 \\
\hline & $\mathrm{F}$ & & & & 10 & & 10 \\
\hline \multirow{3}{*}{$\begin{array}{c}\mathrm{CBi}+ \\
\text { High body weight } \\
\text { Long tail }\end{array}$} & M & & & & & 10 & 10 \\
\hline & $\mathrm{F}$ & & & & & 10 & 10 \\
\hline & & & & & & Total & 100 \\
\hline
\end{tabular}

The two first principal components (PC1 and PC2) account for $83.3 \%$ and $81 \%$ of morphometric variation in each gender. The remaining components define particular processes of this bone and account for the residual variance. All PC1eigenvectors were 
negative, meanwhile, in PC2, eigenvectors of width measurements were positive whereas those related with length measurements were negative. $\mathrm{CBi}$ - mice, selected for low body weight and short tail had the shortest femurs whereas $\mathrm{CBi} / \mathrm{L}$ mice, selected for low body weight and long tail, had the thinnest ones. $\mathrm{CBi} / \mathrm{C}$ mice had shorter and wider femurs than $\mathrm{CBi} / \mathrm{L}$, and $\mathrm{CBi}+$ femurs were wider and larger than $\mathrm{CBi}$ - ones. Genotypes selected for high body weight $(\mathrm{CBi}+$ and $\mathrm{CBi} / \mathrm{C})$ also differed in femur length and width (shorter and wider in $\mathrm{CBi} / \mathrm{C}$ ). The same was true for genotypes selected for low body weight as $\mathrm{CBi} / \mathrm{L}$ had larger and thinner bones than $\mathrm{CBi}$ -

Table 5

Femur length (mean \pm standard error) in male and female mice selected for body conformation and their reciprocal crosses (Mittelwert und Standardfehler der Femurlänge der Mäuse beider Geschlechter nach Selektion auf Körperproportion und reziproken Kreuzungen)

(a) Agonistic selection

\begin{tabular}{cccccccccc}
\hline \multicolumn{3}{c}{ Males } & \multicolumn{9}{c}{ Females } \\
CBi- & $-\mathrm{x}+$ & $+\mathrm{x}-$ & $\mathrm{CBi}+$ & $\begin{array}{c}\mathrm{H}^{1} \\
(\%)\end{array}$ & $\mathrm{CBi}-$ & $-\mathrm{x}+$ & $+\mathrm{x}-$ & $\mathrm{CBi}^{+}$ & $\begin{array}{c}\mathrm{H}^{1} \\
(\%)\end{array}$ \\
\hline $14.56 \mathrm{a}$ & $16.22 \mathrm{~b}$ & $16.06 \mathrm{~b}$ & $16.72 \mathrm{c}$ & $3.2 *$ & $15.34 \mathrm{a}$ & $17.19 \mathrm{~b}$ & $17.16 \mathrm{~b}$ & $17.63 \mathrm{c}$ & $4.2 *$ \\
\pm 0.070 & \pm 0.058 & \pm 0.066 & \pm 0.087 & & \pm 0.075 & \pm 0.051 & \pm 0.077 & \pm 0.093 & 4.2 \\
\hline
\end{tabular}

(b) Antagonistic selection

\begin{tabular}{cccccccccc}
\hline \multicolumn{3}{c}{ Males } & \multicolumn{1}{c}{ Females } \\
$\mathrm{CBi} / \mathrm{L}$ & $\mathrm{L} \mathrm{x} \mathrm{C}$ & $\mathrm{Cx} \mathrm{L}$ & $\mathrm{CBi} / \mathrm{C}$ & $\begin{array}{c}\mathrm{H}^{1} \\
(\%)\end{array}$ & $\mathrm{CBi} / \mathrm{L}$ & $\mathrm{L} \mathrm{x} \mathrm{C}$ & $\mathrm{C} \mathrm{x} \mathrm{L}$ & $\mathrm{CBi} / \mathrm{C}$ & $\begin{array}{c}\mathrm{H}^{1} \\
(\%)\end{array}$ \\
\hline $16.84 \mathrm{a}$ & $16.87 \mathrm{a}$ & $17.17 \mathrm{~b}$ & $16.73 \mathrm{a}$ & \multirow{2}{*}{$1.4^{*}$} & $16.98 \mathrm{a}$ & $17.56 \mathrm{~b}$ & $17.54 \mathrm{~b}$ & $17.18 \mathrm{a}$ & $2.8^{*}$ \\
\pm 0.082 & \pm 0.067 & \pm 0.068 & \pm 0.062 & & \pm 0.065 & \pm 0.083 & \pm 0.090 & \pm 0.061 & \\
\hline
\end{tabular}

(c) Directional selection for low body weight

\begin{tabular}{|c|c|c|c|c|c|c|c|c|c|}
\hline \multicolumn{5}{|c|}{ Males } & \multicolumn{5}{|c|}{ Females } \\
\hline CBi- & $-x L$ & L x - & $\mathrm{L}$ & $\begin{array}{l}\mathrm{H}^{1} \\
(\%)\end{array}$ & - & $-x L$ & $\mathrm{~L} x-$ & $\mathrm{L}$ & $\begin{array}{l}\mathrm{H}^{1} \\
(\%)\end{array}$ \\
\hline $\begin{array}{l}14.56 \mathrm{a} \\
\pm 0.070\end{array}$ & $\begin{array}{l}16.12 \mathrm{~b} \\
\pm 0.033\end{array}$ & $\begin{array}{l}15.83 \mathrm{c} \\
\pm 0.057\end{array}$ & $\begin{array}{l}16.84 \mathrm{~d} \\
\pm 0.082\end{array}$ & $1.8^{*}$ & $\begin{array}{l}15.34 \mathrm{a} \\
\pm 0.075\end{array}$ & $\begin{array}{c}16.75 \text { b,c } \\
\pm 0.042\end{array}$ & $\begin{array}{l}16.53 \mathrm{c} \\
\pm 0.067\end{array}$ & $\begin{array}{l}16.98 \mathrm{~b} \\
\pm 0.065\end{array}$ & $3.0^{*}$ \\
\hline
\end{tabular}

(d) Directional selection for high body weight

\begin{tabular}{|c|c|c|c|c|c|c|c|c|c|}
\hline \multirow[b]{2}{*}{$\mathrm{CBi} / \mathrm{C}$} & \multicolumn{4}{|c|}{ Males } & \multicolumn{5}{|c|}{ Females } \\
\hline & $\mathrm{Cx}+$ & $+x C$ & $\mathrm{CBi}^{+}$ & $\begin{array}{c}\mathrm{H}^{1} \\
(\%)\end{array}$ & $\mathrm{CBi} / \mathrm{C}$ & $\mathrm{Cx}+$ & $+x C$ & + & $\begin{array}{c}\mathrm{H}^{1} \\
(\%) \\
\end{array}$ \\
\hline $\begin{array}{r}16.73 a \\
\pm 0.062\end{array}$ & $\begin{array}{l}17.04 \mathrm{~b} \\
\pm 0.064\end{array}$ & $\begin{array}{l}17.20 \mathrm{~b} \\
\pm 0.092\end{array}$ & $\begin{array}{l}16.72 \mathrm{a} \\
\pm 0.087\end{array}$ & $2.4^{*}$ & $\begin{array}{l}17.18 \mathrm{a} \\
\pm 0.061\end{array}$ & $\begin{array}{l}17.69 \mathrm{~b} \\
\pm 0.093\end{array}$ & $\begin{array}{l}17.67 \mathrm{~b} \\
\pm 0.078\end{array}$ & $\begin{array}{l}17.63 \mathrm{~b} \\
\pm 0.093\end{array}$ & $1.6^{*}$ \\
\hline
\end{tabular}

(e) Directional selection for short tail (skeleton) length

\begin{tabular}{cccccccccc}
\hline \multicolumn{3}{c}{ Males } & \multicolumn{1}{c}{ Females } \\
CBi- & $-\mathrm{x} \mathrm{C}$ & $\mathrm{Cx}-$ & $\mathrm{CBi} / \mathrm{C}$ & $\begin{array}{c}\mathrm{H}^{1} \\
(\%)\end{array}$ & $\mathrm{CBi}-$ & $-\mathrm{x} \mathrm{C}$ & $\mathrm{C} \mathrm{x}-$ & $\mathrm{CBi} / \mathrm{C}$ & $\begin{array}{c}\mathrm{H}^{1} \\
(\%)\end{array}$ \\
\hline $14.56 \mathrm{a}$ & $16.04 \mathrm{~b}$ & $16.19 \mathrm{~b}$ & $16.73 \mathrm{c}$ & \multirow{2}{*}{$3.0 *$} & $15.54 \mathrm{a}$ & $16.84 \mathrm{~b}$ & $16.87 \mathrm{~b}$ & $17.18 \mathrm{c}$ & $3.0 *$ \\
\pm 0.062 & \pm 0.043 & \pm 0.036 & \pm 0.062 & & \pm 0.075 & \pm 0.033 & \pm 0.066 & \pm 0.061 & $3.0^{*}$ \\
\hline
\end{tabular}

(f) Directional selection for long tail (skeleton) length

\begin{tabular}{cccccccccc}
\hline \multicolumn{3}{c}{ Males } & \multicolumn{9}{c}{ Females } \\
$\mathrm{CBi} / \mathrm{L}$ & $\mathrm{L} \mathrm{x}+$ & $+\mathrm{x} \quad \mathrm{L}$ & $\mathrm{CBi}+$ & $\begin{array}{c}\mathrm{H}^{1} \\
(\%)\end{array}$ & $\mathrm{CBi} / \mathrm{L}$ & $\mathrm{L} \mathrm{x}+$ & $+\mathrm{x} \mathrm{L}$ & $\mathrm{CBi}^{+}$ & $\begin{array}{c}\mathrm{H}^{1} \\
(\%)\end{array}$ \\
\hline $16.84 \mathrm{a}$ & $16.81 \mathrm{a}$ & $16.99 \mathrm{a}$ & $16.72 \mathrm{a}$ & $0.7^{\mathrm{ns}}$ & $16.98 \mathrm{a}$ & $17.50 \mathrm{~b}$ & $17.82 \mathrm{~b}$ & $17.63 \mathrm{~b}$ & $2.1^{*}$ \\
\pm 0.082 & \pm 0.080 & \pm 0.086 & \pm 0.087 & & \pm 0.065 & \pm 0.095 & \pm 0.098 & \pm 0.093 & \\
\hline
\end{tabular}

a,b Values with different letter differ at least at 0.05 level; Sample size: 14 animals per group; In hybrids the first genotype denotes the maternal line; ${ }^{1}$ Heterosis $\left({ }^{*}\right.$ significant $-{ }^{\text {ns }}$ non significant) 
Table 4 presents the result of a classification analysis by means of discriminant functions. Female and male mice of each genotype were correctly identified.

\section{Experiment II}

Femur length for all reciprocal crosses between the four selected lines can be seen in Table 5.

Sexual dimorphism (female $>$ male) in femur length was observed in all genotypes. Reciprocal crosses involving CBi- mice (Table 5 a, c and e), the only short femur genotype, and any of the other three long femur genotypes $(\mathrm{CBi} / \mathrm{C}, \mathrm{CBi} / \mathrm{L}$ or $\mathrm{CBi}+$ ) showed dominant deviations towards long femur values, irrespective of the long femur genotype used. Reciprocal crosses between selected lines with long femurs involving $\mathrm{CBi} / \mathrm{C}$ mice as parental genotype (Table $5 \mathrm{~b}$ and d) showed heterosis with an overdominant effect. Finally, reciprocal hybrids between $\mathrm{CBi} / \mathrm{L}$ and $\mathrm{CBi}+$ mice (Table $5 \mathrm{f}$ ) did not differ in their average femur length neither between them nor from each parental line. The same result was observed in both genders.

\section{Discussion}

Historically, genetic monitoring methods used for inbred laboratory rodents had included skin grafting, test mating for hidden coat color genes, immunological markers, biochemical markers, and mandible analysis. Notwithstanding nowadays the availability of molecular procedures make possible an accurate genetic identification, the analysis of bone morphometry is still important as a mean of phenotyping and discriminating mouse lines prior to their use in crosses designed to generate segregating F2 populations which maximize linkage disequilibrium among QTLs and molecular markers exhibiting classical Mendelian segregation.

Mice of the CBi stock were selected simultaneously for either high or low body weight and either short or long skeletal length. This genetic strategy led to morphological distinct animals, with different body conformations (CBi/C: compact; $\mathrm{CBi} / \mathrm{L}$ : longilineal; $\mathrm{CBi}+$ : large; $\mathrm{CBi}-:$ small), which modified their skeleton in response to differences in the biomass sustained. Univariate analysis of a set of mandible measurements previously reported (DI MASSO et al., 1997c) evinced some particularities in the mandible morphogenesis of these lines. Using mandible measurements, lines could be identified by a discriminate analysis, with low probability of wrong discrimination. Although the mandible is an efficient tool for identifying lines of rats, mice and rabbits it is not involved in the support of the soft tissues and like most of the craniofacial bones it develops through membranous ossification. Therefore, it seemed relevant to choose a long bone, like the femur, with endochondral ossification, more closely related to the selective procedure as the discriminative criterion for these genotypes. In accordance with the results described for the mandible, all PC1 eigenvectors yielded by the principal component analysis were negative and so, they could be interpreted as a size factor. In PC2, eigenvectors of width measurements were positive whereas those related with length measurements were negative, so it can be interpreted as a form factor (JOLICOEUR and MOSIMAN, 1960).

Besides the previously reported usefulness of morphometric mandible analysis, the results herein described show that the morphometric profile of the femur is a reliable and more accurate means of identifying these inbred strains of mice selected for different body conformations. This fact is related to a particular response to artificial 
selective pressure in each line which depends on a specific combination of body weight and tail (skeleton) length values.

A QTL analysis can allow us to address specific questions concerning genetic architecture, such as the number of loci potentially affecting the trait, the distribution of gene effects, and the underlying patterns of gene action, including additivity, dominance, gender-specificity, epistasis and pleiotropy. In this sense, results from Experiment II suggest that those genotypes selected for long tail (skeleton) irrespective if they were simultaneously selected for high $(\mathrm{CBi}+)$ or low $(\mathrm{CBi} / \mathrm{L})$ body weight enlarged their femurs by using the same source of genetic variation for the trait, as neither both of them nor their reciprocal hybrids differ in their mean femur length. A different scenario emerge when $\mathrm{CBi} / \mathrm{C}$ was used as parental line. Notwithstanding these mice also enlarged their femurs and, as a consequence, do not differ in femur length from $\mathrm{CBi}+$ and $\mathrm{CBi} / \mathrm{L}$ mice, this response was achieved when short tail (skeleton) length was selected. When $\mathrm{CBi} / \mathrm{C}$ mice were crossed to either $\mathrm{CBi} / \mathrm{L}$ or $\mathrm{CBi}+$, the other two lines showing long femurs, an overdominance effect was evident. This response could be interpreted as the result of combining in the same animal different genes for femur length: those provided for the $\mathrm{CBi} / \mathrm{C}$ parent and those provided for the $\mathrm{CBi}+$ or the $\mathrm{CBi} / \mathrm{L}$ parent. So, it could be argued that genes involved in enlargement of the femur when the selective criterion acts against the function of the skeleton as a scaffold for the soft tissues are different from those genes responsible of the same response when that change is compatible with the aforementioned function. Finally, when $\mathrm{CBi}$ - was crossed to the other three selected lines $(\mathrm{CBi} / \mathrm{L}$ or $\mathrm{CBi} / \mathrm{C}$ or $\mathrm{CBi}+$ ), genes for short femur length always showed recessiveness. irrespective of the line of origin of the genes for long femur.

Although targeted gene deletions (gene knockouts) and transgenics jointly with congenic lines generated by introgressing a chromosomal region in a particular genetic background offer a wide spectrum of models to study individual genes and gene products, they require previous knowledge about the genes associated with the phenotype under study. On the contrary, anonymous genes underlying complex traits can be identified by positional cloning based solely on their position in the genome without any knowledge about their functions (CORVA and MEDRANO, 2001). In this sense, lines generated by long-term artificial selection are valuable resources to create suitable mapping populations. As the number of QTLs mapped in a particular study is limited to those at which different alleles are fixed in the two parental strains (MACKAY, 2001), and this appears to be the case with the genotypes herein described, it could be concluded that this animal model could be an useful resource for mapping femur growth genes increasing our understanding of the signalling pathways and the transcription factors that control bone development (KRONENBERG, 2003).

\section{Acknowledgements}

We thank Mrs. Fabiana Severino and Miss María Elena Ponte for technical assistance. This research was partly supported by a grant of the Secretaría de Ciencia y Tecnología, Universidad Nacional de Rosario.

\section{References}

BROCKMANN, G.; BUITKAMP, J.; TEUSCHER, F.; BÜNGER, L.; EPPLEN, J.T.; SCHWERIN, M.:

Multilocus fingerprint bands are associated with the growth performance in selected mouse lines: linkage analysis in reference families. Arch. Tierz., Dummerstorf 39 (1996), 477-487 
BÜNGER, L.; LAIDLAW, A.; BULFIELD, G.; EISEN, E.J.; MEDRANO, J.F.; BRADFORD, G.E.; PIRCHNER, F.; RENNE, U.; SCHLOTE, W.; HILL, W.G.:

Inbred lines of mice derived from long-term growth selected lines: unique resources for mapping growth genes. Mamm. Genome 12 (2001), 678-86

BÜNGER, L.; OTT, G.; VARGA, L.; SCHLOTE, W.; RENNE, U.; WILLIAMS, J.L.; HILL, W.; REHFELDT, $\mathrm{CH}$ :

Marker assisted introgression on the Compact mutant myostatin allele: Mstn ${ }^{\text {Cmpt-dllAbc }}$ into a mouse line with extreme growth: effects on body composition, muscularity and skeletal muscle cellularity. Arch. Tierz., Dummerstorf 48 (2005) Special Issue, 88-97

CORVA, P.M.; MEDRANO, J.F.: Quantitative trait loci (QTLs) mapping for growth traits in the mouse: A review. Genet. Sel. Evol. 33 (2001), 105-32

DAS, P.; BROCKMANN, G.; MEYER, L.; RENNE, U.; FREYER, G.; SCHWERIN, M.:

The effect of a restricted region of chromosome 11 on body weight in mice under special consideration of the growth hormone gene locus. Arch. Tierz., Dummerstorf 39 (1996), 185-194

DI MASSO, R.J.; CELORIA, G.C., FONT, M.T.:

Morphometric traits and femoral histomorphometry in mice selected for body conformation. Bone Miner. 15 (1991), 209-18

DI MASSO, R.J., CELORIA, G.C., FONT, M.T..

Morphometric skeletal traits, femoral measurements and bone mineral deposition in mice with agonistic selecction for body conformation. Bone 22 (1998), 539-43

DI MASSO, R.J.; FONT, M.T.; CAPOZZA, R.F.; DETARSIO, G.; SOSA, F.; FERRETTI, J.L.: Long-bone biomechanics in mice selected for body conformation. Bone 20 (1997a), 539-45

DI MASSO, R.J.; SILVA, P.S.; FONT, M.T.:

Muscle-bone relationships in mice selected for different body conformations. J. Musculoskel. Neuron. Interact. 4 (2004), 41-47

DI MASSO, R.J.; ZERPA, C.M.; FONT, M.T.:

Modification of the body sustentation system in mice selected for different body conformations. Mendeliana 12 (1997b), 111-22

DI MASSO, R.J.; ZERPA, C.M.; FONT, M.T.:

Identification of mouse lines selected body conformation using the morphometric profile of the mandible. Mouse Genome 95 (1997c), 882-885

FESTING, M.F.W.:

Mouse strain identification. Nature 238 (1972), 351-352

FISLER, J.S.; WARDEN, C.H.:

Mapping of mouse obesity genes: A generic approach to a complex trait. J. Nutr. 127 (1997), 909S-16S

HALEY, C.S.; KNOTT, S.A.; EISEN, L.M.:

Mapping quantitative trait loci in crosses between outbred lines using least squares. Genetics 136 (1994), 1195-1207

HINRICHSEN, L.; MANA, D.; DI MASSO, R.; FONT, M.T.:

Effect of disruptive selection for body conformation on age variations of femoral morphometric traits in mice. Arch. Tierz., Dummerstorf 42 (1999), 201-209

JOLICOEUR, P.; MOSIMAN, J.E.:

Size and shape variation in the painted turtle. A principal component analysis. Growth 24 (1960), 339354.

KRONENBERG, H.M.:

Developmental regulation of the growth plate. Nature 423 (2003), 332-336.

LOVELL, D.; JOHNSON, F. M.:

Quantitative genetic variation in the skeleton of the mouse. I. Variation between inbred strains. Genet. Res. 42 (1983), 169-82

MACKAY, T.F.C.

The genetic architecture of quantitative traits. Annu. Rev. Genet. 35 (2001), 303-309

MIELENZ, N.; SCHÜLER, L.:

Nutzung von QTL-Information bei der Langzeitselektion. Arch. Tierz., Dummerstorf 45 (2002), 87-97

ROSOCHACKI, S.J.; WIRTH-DZIECIOLOWSKA, E.; ZIMOWSKA， M.; SAKOWSKI, T.; POLOSZYNOWICZ, J.; JUSZZUK-KUBIAK, E.; GAJEWSKA, M.:

Skeletal muscle and liver protein degradation in mice divergently selected for low and high body weight over 108 generations. Arch. Tierz., Dummerstorf 48 (2005), 505-517

SHERIDAN, A.K.:

Crossbreeding and heterosis. Animal Breeding Abstracts 49 (1981), 131-44

SOKAL, R.R.; ROHLF, F.J.:

Biometry. The principles and practice of statistics in biological research. W.H. Freeman and Co., San 
Francisco, CA, 1969.

TALBOT, C.J.; NICOD, A.; CHERNY, S.S, FULKER, D.W., COLLINS, A.C., FLINT, J.

High-resolution mapping of quantitative trait loci in outbred mice. Nat. Genet. 21 (1999), 305-08

TATSUOKA, M.:

Multivariate analysis. Techniques for educational and psychological research. John Wiley and Sons, Inc., New York, NY; 1971.

WALTER, E.; BUTLER-WEMKEN I., v.; PIRCHNER, F.:

Anatomische Auswirkungen einer Selektion auf verschiedenes Körpergewicht in zwei Mäuselinien. Arch. Tierz. Dummerstorf 36 (1993), 85-93

ZENG, Z.B.; KAO, C.H.; BASTEN, C.J.:

Estimating the genetic architecture of quantitative traits. Genet. Res. 74 (1999), 279-89

Received: 2006-05-19

Accepted: 2007-02-22

Authors' address

Dr. RICARDO J. DI MASSO, CESAR M. ZERPA, M.Sc., Ing. Agr. PATRICIA S. SILVA, Statistician MARIA TERESA FONT

Instituto de Genética Experimental

Facultad de Ciencias Médicas

Universidad Nacional de Rosario

Santa Fe 3100

2000 ROSARIO, ARGENTINA 